\title{
A Descriptive Study on Prescription audit of prescribing patterns in primary, secondary, tertiary healthcare facilities in India
}

\author{
*Debraj Mukhopadhyay ${ }^{1}$, Dr. Vibhor Dudhraj², Dr. Dattatreya Mukherjee ${ }^{3}$ \\ ${ }^{1,2}$ Department of Public Health, School of Allied Health Sciences, Delhi \\ Pharmaceutical Sciences and Research University (DPSRU), Govt. of NCT Delhi, \\ New Delhi - 110017 \\ ${ }^{2}$ Jinan University, P.R China
}

\section{Introduction}

Prescription audit is a tool as well as a technique and its application is science as well as an art. Quality of life can be improved by enhancing the standards of medical treatment and that can only be assessed by prescription audit, because it is based on documented evidences to support diagnosis, treatment and justified utilization of hospital facilities. Effective prescription audit is important for health professionals, health service managers, patients, and the public. It supports health professionals in making sure their patients receive the best possible care. It can inform health service managers about the need for organizational changes, or new investment to support health professionals in their practice. Prescription audit is a quality improvement process that seeks to improve patient care(1).

Antibiotic resistance is a global problem which is threatening, particularly in developing countries where the infectious disease burden is more and cost constrains the replacement of older antibiotics with newer which are more expensive ones. Management of common and lethal bacterial infections has been critically compromised by the appearance and rapid spread of antibiotic - resistant bacteria(2).

Antibiotics were once considered 'miracle drugs' and have been used for decades to effectively treat a variety of bacterial infections. Unfortunately, widespread use and misuse worldwide have led to the emergence of 'super bugs' and other drug-resistant bacteria. The bacterial disease burden in India is among the highest in the world(3); consequently, antibiotics will play a critical role in limiting morbidity and mortality in the country. As a marker of disease burden, pneumonia causes an estimated 410,000 deaths in India each year, and it is the number-one killer of children (2). Many of these deaths occur because patients do not have access to life-saving antibiotics when and where these are needed. Antibiotics are used in situations where these cannot be expected to improve the patient's condition, particularly as treatment for the common cold and uncomplicated cases of diarrhea "Drug selection pressure" is the single most important factor in the evolution of drug resistance in bacteria. Antibiotic use has been increasing steadily in recent years. Between 2005 and 2020, the units of antibiotics sold increased by about 40\%. Increased sales of cephalosporin were particularly striking, with sales (in units sold) increasing 
by $60 \%$ over that five-year period, but some increase was seen in most antibiotic classes. The increased resistance is a result of many factors, but the foremost cause is the overall volume of antibiotic consumption. The fact that antibiotic use is increasing is not, itself, indicative of a problem, but evidence from studies of prescribing patterns suggests that antibiotics are often used in inappropriate ways (4). Components of prescription; Superscription, it includes patient's name, address, age, date of birth, date of admission, weight and date of prescription writing and the Prescription Symbol $(\mathrm{R})(5,6,7,8,9,10,11)$. Inscription, it is the body of prescription. It is written just below and right to superscription. It consists of drug name and dose form (e.g. tablet, lotion, suspension). It is always recommended to use generic drug names than brand names to avoid confusion as brand names often differ widely in different countries for the same medicine. Besides, many brand name drugs have less expensive generic drug substitutes that are therapeutically and biochemically equivalent 1 The use of apothecary or avoirdupois units and symbols of measure such as pints (O), drams (3), and grains (gr) is largely replaced by metric equivalents (12). Subscription, it should be written a line below the inscription and includes prescriber`s direction to pharmacist, that is, dose form and total amount to be dispensed.

Mistakes, where the plan itself is wrong e.g. writing 50mg of a drug not knowing the usual dose is $5 \mathrm{mg}$. One of the primary communication links between prescriber, pharmacist and the patient is complete, safe, and accurate prescription. Completion of all 'essential elements' using the standardized format of a prescription will assure that it is accurately interpreted and not subject to alteration (13).

Impact of irrational use of drugs $(14,15)$, this can be seen in many ways:

I. Reduction in the quality of drug therapy leading to increased morbidity and mortality.

II. Waste of resources leading to reduced availability of other vital drugs and increased costs.

III. Increased risk of unwanted effects such as adverse drug reactions and the emergence of drug resistance.

IV. Psychosocial impact, such as when patients come to believe that there is "a pill for every ill", which may cause an apparent increased demand for drugs.

Error can arise from any step of prescribing such as the choice of drug, dose, route of administration and wrong frequency or duration of treatment. Inaccuracy in writing and poor legibility of handwriting or incomplete writing of a prescription can lead to misinterpretation, thus leading to errors in dispensing and administration. Health professionals must take account of appropriateness, effectiveness, side effects, contraindications and cost when prescribing any medicine. Special care and precautions are needed in prescribing the drugs with abuse potentials to avoid their misuse. Irrational prescribing may account to professional and legal threat to a prescriber if done injudiciously as it indicates the prescriber's responsibility towards clinical care and safety of patient. Every country has its own standards and regulations for prescription writing. There is no global standard for prescription writing which may suggest a need for 
introduction of universal guidelines. This desk review was in the purpose to know the antibiotic purpose seeing many articles.

\section{Objective:}

To review prescription audit of prescribing patterns in primary, secondary, tertiary healthcare facilities in India.

\section{Methodology:}

To address the objective of this review, literature review was used to first identify appropriate research and review papers which accessed the prescribing patterns from primary, secondary and tertiary healthcare facilities in India. Literature review was used to address all the objectives as it led to comprehensive findings in the desired field area with substantial emphasis on Prescription Audit. It also led to identification of the recent work done in a more systematic manner.

Comprehensive research was conducted using web-based search engines like Google scholar, Science Direct, Pub Med / NCB, IMS health using a combination of search string: prescription audit, prescription consumption, prescribing patterns, and importance of rational prescription writing in Govt. or Private Hospitals in India. The preliminary search on Pub Med was conducted using mesh terms like "prescription audit of prescribing patterns in primary, secondary, tertiary healthcare facilities in India". The search yielded 65papers between the time periods 2008-2019 which were shortlisted on the basis of abstract of these studies. To further filter the studies, PICO inclusion and exclusion criteria were set up as demonstrated in Table No.01

Table No. 01 - Inclusion and Exclusion criteria for study

\begin{tabular}{|l|l|l|}
\hline & Inclusion Criteria & Exclusion Criteria \\
\hline Interventions & Quality, Importance & \\
\hline Comparison & Pre and post interventions & \\
\hline
\end{tabular}




\begin{tabular}{|l|l|l|}
\hline Area of Study & $\begin{array}{l}\text { Based on prescription audit in primary, } \\
\text { secondary and tertiary care government and } \\
\text { private hospitals in India. }\end{array}$ & $\begin{array}{l}\text { The studies conducted outside } \\
\text { from India. }\end{array}$ \\
\hline Study Design & Descriptive, correlation, review & \\
\hline
\end{tabular}

Table 02: Study design in flow chart

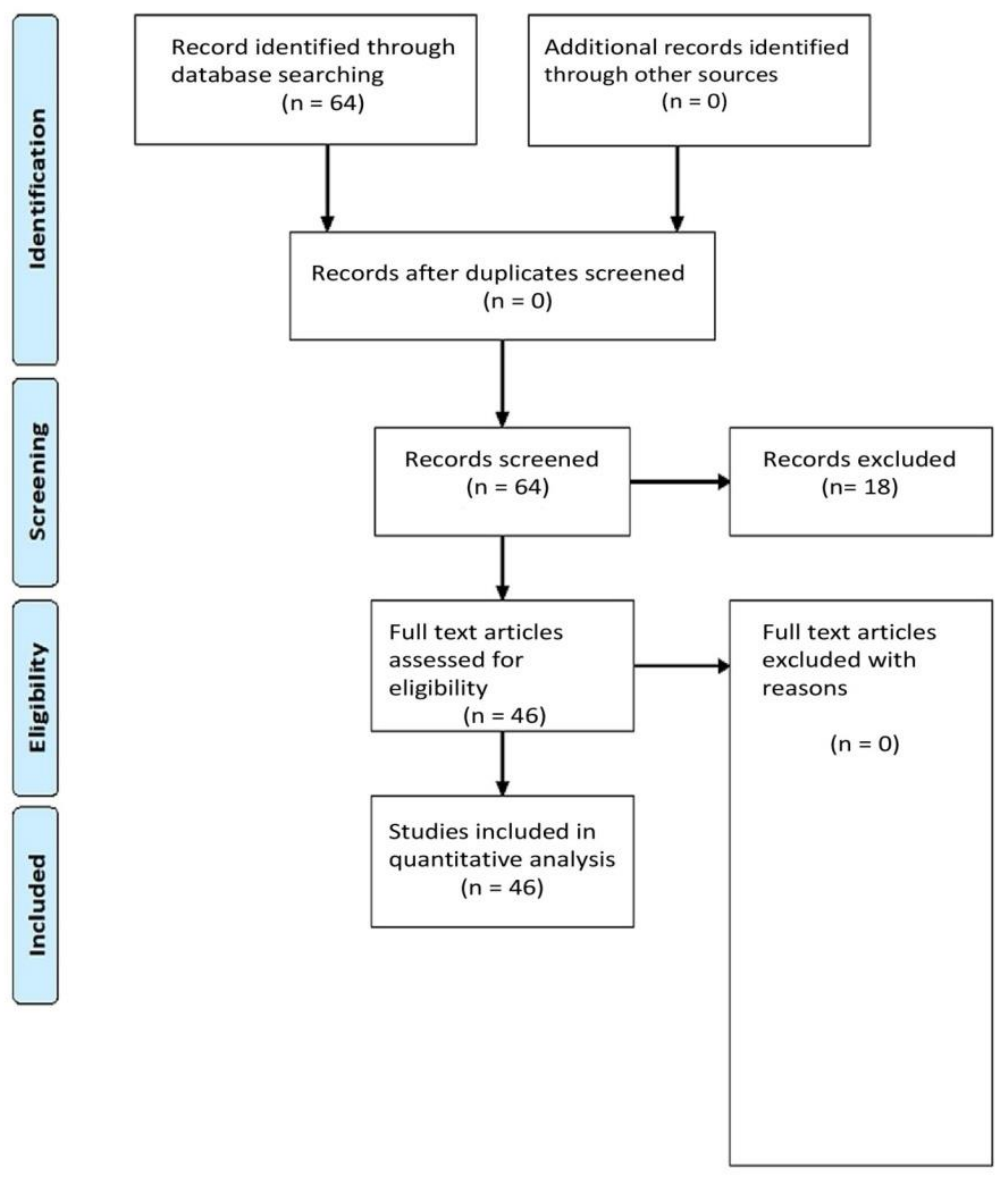




\section{Review of Literatures:}

Amit Chakrabarti et al., states a scrutiny of the relevant product communications indicates that there is little or no clinical evidence to substantiate this claim. Despite this, FDCs have gained acceptance among physicians, as is evident by the growing number of such prescriptions.

Kandula PK et al., states that the results obtained from the study shows the prevailing prescribing habits at their institution. Much no. of prescribing trends from this study, are a cause of concern and need attention, here its reveal that the auditing of prescription in terms of rationality, it remains poor. The value of such audits in generating and testing hypotheses on inappropriate prescribing will definitely create an intervention to improve prescribing habits.

Nihar R. Biswas et al., indicated an awareness of poly-pharmacy but a high incidence of common prescription writing errors such as not recording the duration of therapy, frequency of administration and dosage form. Moreover prescribing by generic name was also low.

M.J. Kshirsagar et al., states the frequency with which combinations of antimicrobials were prescribed in our study is high and greater reflection is needed by doctors before writing a prescription for two or more antimicrobials. Prescriptions for tonics and vitamins increased with the number of drugs per prescription, and contributed to the polypharmacy and increased costs.

D. Padmini Devi et al., states Insulin was highly preferred over oral hypoglycaemic drugs. 4. the majority of study patients were type 2 diabetics. 5. Hypertension and diabetic retinopathy were the two associated major co-morbid factors of DN seen in $100 \%$ of patients. The majority of patients $(93 \%)$ were in late stage of DN (stage 4 or 5) with chronic renal failure. 6. The average number of drugs per prescription was 7.4 only $12 \%$ of the drugs were prescribed by generic name around $32 \%$ of the total numbers of drugs were given as injections, which are a commonly overused and costly form of drug therapy.

M.V. SRISHYLA et al., states in the case of NSAIDs was the frequency of prescribing significantly more in females than in males. The prescribing frequency of NSAIDs was higher in females than in males $(p=0.03)$. Frequency of prescribing of CVS drugs increased with age for both sexes $(\mathrm{P}<0.0001)$. In the 31-40 age group, prescribing frequency of CVS drugs was significantly higher in males than in females $(p=0.003)$ while it was vice versa with NSAlDs $(p=$ 0.04). There was significant though irregular difference in the prescribing frequency of NSAlDs $(\mathrm{p}=0.05)$ and of CNS drugs $(\mathrm{p}=0.0003)$ in males of different age groups. In males, the prescribing frequency of APD drugs was significantly lower in the below-20 age groups as compared with the above-20 age groups $(\mathrm{p}=0.02)$.

R. K. Rishi et al., states that the survey reveals a high prevalence of drug exposure for the patients, indiscriminate use of NSAIDs, antibiotics and vitamins. The most commonly used tool in the prescription audit is the measurement of the average number of drugs prescribed per prescription. 
Hanumantha Rao et al., 2011 states that about 77 percent of the prescriptions contained only one diagnosis. The average number of drugs per prescription was 3.1. About 60 percent of the drugs were prescribed by generic names and about 23 percent of the prescribed drugs were in combination. About 25 percent of the prescriptions contained at least one injection, while 35 percent contained at least one antibiotic. In 16 percent of the prescriptions a vitamin or tonic was prescribed. About 46 percent of the single ingredient formulations were as per the WHO 2003, Essential Medicines List (EML). Based on the findings of the Prescription Audit an EML was prepared for each category of the secondary level hospitals, for use in the OPD. However, all details namely (a) strength, (b) dose, and (c) number of days were mentioned only in 38.8 percent of the tablets, in 50.1 percent of the capsules, and in 16.1 percent of the injections. By default in project hospitals drugs were given for three days, unless the prescription mentioned otherwise. 2. About 31 percent of the prescriptions contained four or more drugs. By comparing with the STGs for diseases for which such prescriptions were made, they could be justified or categorized as irrational prescriptions. 3. Still about 40 percent of the doctors used brand names. The reasons for using brand names need to be understood (such as comfort ability with brand names, opinion that generic drugs are of low quality, etc.) and addressed with appropriate interventions.

Deepa Sunny et al., 2019 states that five hundred scripts comprising of 1,661 drugs were analyzed. The average number of drugs per prescription was three. The study encompassed $52 \%$ males and 48\% females. Most patients were from the age group of 41-60 years. Only 3.6\% (18) of medications were prescribed by generic names. Patients received 9\% (145) medications contained in Essential Drug List and prescriptions containing antibiotics were 19\% (97). Majority of the prescriptions were from Orthopaedics (18.6\%) tailed by General Medicine (15.8\%). Consecutively Analgesics (12.7\%) were the most commonly prescribed drug class among which Diclofenac and Paracetamol+Tramadol were usually prescribed, this was tailed by gastrointestinal medicines (11.7\%). Prescription audit can be helpful to plan appropriate intervention to ensure the rational drug therapy and to evaluate the existing drug use pattern. It also reflects the perspectives of current prescribing pattern in hospitals.

Shahid Hussain et al., 2016 states that Most common diagnosis was diseases of respiratory system (28.3\%). Among therapeutic classification, drugs most commonly used were antimicrobials (20.87\%) and most common antimicrobial was penicillin at the rate of $31.7 \%$. Among penicillin combination of amoxycillin and clavulanic acid was most commonly prescribed. A total of $96.3 \%$ encounters were having single antimicrobials. Of all, 61.8\% prescriptions contained fixed-dose combinations in which combination of expectorants and cough suppressants was most common. Many of the prescribing trends from this study are a cause of concern and need attention. The value of such audits in generating and testing hypothesis on inappropriate prescribing will definitely create an intervention to improve prescribing habits and ultimately patient care will be improved. 
Dr Debasis Bandyopadhyay et al., 2014 states that general medicine OPD contributing 45.38\% patients with the most frequent diagnosis was the disease of the gastro-intestinal system (ICD 10 code- k00-k99) at rate of $12.33 \%$. 19\% of prescription contain more than single diagnosis with total numbers of drugs prescribed were 18559. Most frequent antibacterial agent was fluoroquinolones subgroup (ATC code-JOIMA) at the rate of $7.78 \%$. In this study it was observed that in $91.33 \%$ of prescription weight is not written, while in-appropriate drugs prescribed in 52.99\% of prescription. Average number of drugs per encounter was 4.4; percentage of drugs prescribed by generic name was only $20.99 \%$, while percentage of encounters with an antibiotic prescribed was $28.89 \%$, \& percentage of encounters with an injection prescribed was $28.99 \%$. Percentage of drugs prescribed from essential drugs list or formulary was only $60.98 \%$, and overall illegibility of prescription was $22.99 \%$. The results of this study show the prevailing prescribing habits at our institution. This study reveals that the auditing of prescription in terms of rationality, it remains poor. The value of such audits in generating and testing hypotheses on inappropriate prescribing will definitely create an intervention to improve prescribing habits and ultimately patient care will be improved.

Apollo James et al., 2013 states that out of a total of 360 patients who were taken for study, 210 were from the urban areas and 150 were from the rural areas.48\% women and $52 \%$ of men were taken into the study based on infected system Totally $51.7 \%$ patients got infection in upper respiratory tract, while only 9 patients reported GIT infections in urban areas. While $27 \%$ has the complaints of the GIT in the urban areas. $86 \%$ in rural and $97.1 \%$ in urban were diagnosed by symptoms, $42 \%$ and $84.3 \%$ were diagnosed by the physical examination and least $10 \%$ from rural and $17 \%$ from urban was diagnosed on the basis of lab reports. By these complete observations the study reveals that antibiotics are extensively used in both rural and urban sectors. Irrational usage of antibiotic is a key driver of antimicrobial resistance. Antibiotics are overused particularly for minor infections, misused for self limiting viral infections. However, the effort of collecting this data is only worthwhile if policy makers invest in the intervention urgently needed to improve the use of antibiotics in the community, without such actions increasing antimicrobial resistance will deny future generations the benefit of effective antibiotics to treat common infections.

Muhsina Taskeen et al., 2012 states that Out of 150 prescriptions studied, 83 (55.34\%) belonged to males and the rest $67(44.37 \%)$ to females, giving a male to female ratio of 1:0.80. Anti diabetics $(142,15.58 \%)$ were the most commonly prescribed medicines with metformin being the most prescribed anti diabetic. The medications prescribed by generic names were 92 (10.08\%). $40 \%$ of medicines were prescribed from the WHO essential drug list. 17 (11.3\%) prescriptions had one or more potentially inappropriate medicines from Beer's criteria and in 16 (10.6\%) prescriptions drug - drug interactions were ascertained according to drug - drug interactions of common OTC drugs. Prescribing for the elderly was found to be suboptimal and there was occurrence of inappropriate prescribing. This calls for caution on the part of prescribers and pharmacists alike and also the need for awareness of tools that can be used by 
practitioners for detecting drug therapy problems. More studies are required on the pattern of inappropriate prescribing over a long period of time and on intervention programs to reduce potentially adverse health outcomes in elderly patients most at risk in the area where this study was undertaken.

Bala Sharmin et al., 2014 states that the total number of prescriptions, 46\% prescriptions was containing at least one or more than one antimicrobial agent. Average number of antimicrobials prescribed per prescription was 1.35 . Cotrimoxazole was the most common antimicrobial agent prescribed. Of the total 307 items of prescribed antimicrobials, 57\% were prescribed by proprietary name and $43 \%$ by nonproprietary name. Out of the antimicrobial items prescribed, $44 \%$ were available at the pharmacy of the Urban Health Centre. Fifty eight percent of antimicrobials prescribed were from WHO Essential Drug List. Among the various antimicrobials prescribed at the Urban Health Centre, cotrimoxazole was found to be the most commonly prescribed antimicrobial agent in the year 2012. More than half of the antimicrobials were prescribed by proprietary name. Less than half of the antimicrobials prescribed were available at the Urban Health Centre. Majority of the antimicrobials prescribed were from the WHO Essential Drug List.

Rajneeta Kumari et al., 2008 states that The important components of prescription viz. examination findings, weight of the child, follow up visit and the signatures of the prescribers were absent in the prescriptions at the primary level. Polypharmacy was common $(3.1 \pm 1.6$ drugs per prescription). The prescription of drugs by generic name was low (27.1\%). The prescriptions at the secondary level health facilities were incomplete with respect to mentioning the suffix/prefix of the drug, full name, dose, frequency and strength of the drugs, and directions specifying the route and duration of the treatment. The average cost of drugs/prescription/day in US\$ (Mean, SD) was found to be the highest at the tertiary level $(0.34,0.43)$, which decreased significantly at the primary level health facilities. The pattern of prescription in terms of completeness and rationality was poor. There is an urgent need to improve the standards of drug prescription.

Harmeet Singh Rehan et al., 2002 states that Among the 1457prescriptions, collected, the average number of drugs perprescription was 2.47.The commonest groups of drugs prescribed were antibiotics (33.9\%), analgesics and anti-inflammatories(17.0\%), vitamins (13.0\%), cough syrups(10.5\%)and antihistamines (8.6\%). The use of injectables was uncommon (0.9\%).Most of the drugs prescribed were from the local drugs list of the centre (84.2\%) consisting of 36 drugs. As per Kunin's criteria, 57.9\%of the antibiotics used were appropriate. Interns often forget to write the diagnosis (43\%), signs and symptoms (50.2\%), dosages and frequency of treatment. The results of this study emphasize the need for comprehensive measures including training, information and education as part of the undergraduate curriculum to improve the prescribing behavior of future doctors. 
Kumari Indira K.S et al.,2008 Overall antimicrobial prescription rate was 69.4 per cent (95\% CI 67.1, 71.7). Wide variation was observed (Thiruvananthapuram 47.6\%, Lucknow 81.8\%, Chennai $73.1 \%$ and Vellore 76.5\%). Physicians practicing in rural and public/government settings prescribed antimicrobials more frequently than those in urban and private settings (83.8, $81.9,68.3$ and $68.2 \%$ respectively). Antimicrobials were more frequently prescribed for patients presenting with fever. Highest rate was noticed for children aged between 6 and 18 yr. Patients of the high-income group received antimicrobials more frequently (72.7\%). In both public/government and private settings, for patients who purchased medicines, the rate was higher (82.4 and 68.9\% respectively), vs. those receiving free medicines (70.2 and $46.2 \%$ respectively). Two third of all antimicrobials prescribed were penicillins and co-trimoxazole, and $>40$ per cent of prescriptions from private sector were quinolones and cephalosporins. Our findings showed that prescription of antimicrobials for acute respiratory infections and diarrhoea was extremely common and warrants interventional strategies.

V. Pavani et al.,2012 Out of 677 patients $56.6 \%$ were males and $43.4 \%$ were females with age ranging from 31 years to 80 years.the 203 prescriptions issued by specialist 1 had a mean of 2.0 (27.2\%) drugs per prescription, whereas the 257 prescriptions issued by specialist 2 had 3.2 (43\%) drugs per prescription and by specialist 32.2 (29.8\%) drugs per prescriptions out of 217 prescriptions. This suggests that there were no discernible differences between the prescribing behaviors of physicians of the outpatient department which was not statistically significant $(\mathrm{p}=0.075)$. This study provides important insights in to the drug use patterns in the three outpatient departments. It has helped to identify use of branded drugs by three different physicians which made more economical.

Nitin B. Mali et al., 2018 A total of 200 patients enrolled, among them 119 males and 81 females. There were $12.46( \pm 6.16)$ drugs prescribed per patient, of which $2.38( \pm 1.48)$ were antibacterials. Among the total drug prescribed, $18.49 \%$ were antibacterials and $97 \%$ patients received at least one antibacterial. Ceftriaxone $(49.48 \%)$ was the most commonly prescribed antibacterial, while imipenem (2.58\%) and colistin (2.06\%) use was very low. A total of $80.95 \%$ antibacterials were prescribed by generic name, $94.88 \%$ were administered intravenously, and $80.76 \%$ were prescribed from hospital pharmacy. The averageb length of PICU stay was 6.15 days $( \pm 6.20)$, the average length of antibacterial treatment was 6.08 days $( \pm 6.27)$, and the average length of empirical antibacterial treatment was 5.50 days $( \pm 5.40)$. The cost of antibacterial therapy per patient was Indian rupees $824.64( \pm 235.35)$. In 27 patients, bacterial culture test was positive and of whom 21 received antibacterials as per sensitivity pattern. The use of antibacterials was not indiscriminately high but more prescriptions per sensitivity pattern are required.

G. Mohlala et al.,2010 high mean number of drugs were prescribed per patient (3.2 versus 2.8) in public hospitals and by general practitioners (GPs) respectively and generic prescribing rates were low (45.2\% versus $24.5 \%$ ). The rates of prescribing in public hospitals and by GPs were $8.3 \%$ versus $23.3 \%$ for injections, $68.1 \%$ versus $31.9 \%$ for antibiotics and $92.6 \%$ versus $68.5 \%$ 
for drugs from the essential drugs list. Our findings suggest that drug prescribing by public and private prescribers needs to be regulated closely in South Africa. Use of injections was not found to be a problem in the 2 provinces studied. With regards to the prescribing of generic medicines, all prescribers should be obliged to prescribe generically to give the patient a choice of brand they want to use at the cost that suits them.

DEEPAK G SHEWADE et al., 1998 Among the types of drugs used, antimicrobial agents were prescribed maximum in the government teaching hospital $(26.9 \%)$ as well as by the general practitioners (23.7\%). Analgesics, antiulcer drugs, vitamin preparations were the commonly prescribed agents by the general practitioners while topical medications, analgesics, antihistamines, and bronchodilators were the commonly prescribed drugs by the government doctors. General practitioners prescribed glucocorticoids, anabolic steroids, zinc with vitamins or vitamin $\mathrm{E}$ which were expensive, ineffective or may be harmful. lnjectables were also more frequently prescribed by them. Interestingly the prescriptions of government teaching hospital were more rational since the prescribers used less number of drugs in average and more frequently in their generic names. This study may help to identify the problems involved in therapeutic decision making and improve the prescribing behaviour by planning for an interventional strategy.

Afroz Abidi et al., 2013 Two hundred and thirty seven prescriptions were analyzed. Total no. of drugs in 237 prescriptions were 1001. Therefore average number of drugs/prescription is 4.22. Drugs were prescribed by generic names in $3.79 \%$ of cases, drugs on EDL are only $53.25 \%$ and fixed dose combinations are $26.87 \%$ of total drugs. Dosage forms used were mostly oral $93.51 \%$. Injectables were only $6.19 \%$ and topical forms were least $0.299 \%$. Doctors profile indicates that maximum number i.e. $93.67 \%$ were general practitioners. Basic information of patient was written in $72.57 \%$ prescriptions. Complete diagnoses were written in $70.04 \%$ prescriptions. Only $88.61 \%$ prescriptions were legible and only $76.79 \%$ prescriptions were complete in terms of dose, route, strength, frequency and dosage forms. Disease pattern seen was variable. Diseases of respiratory system were maximum $44.72 \%$ followed by infectious and parasitic diseases - $16.03 \%$ and diseases of digestive system - $13.92 \%$. This study showed that there is scope for improvement in prescribing patterns in areas of writing generic names of drugs, essential drugs, writing legible and complete prescriptions. Polypharmacy was also evident from our study. Establishment and implementation of appropriate clinical guidelines, use of essential medicines list, public education about medicines and regular update to the clinicians will help in implementing the principles of rational pharmaco-therapeutics.

Nishita H. Darji et al., 2015 Three hundred six prescriptions were analysed in which 1986 drugs were prescribed. Mean number of drugs per prescription was $6.49 \%$. In our study, $63.34 \%$ drugs were prescribed by generic names and drugs on NLEM were $73.01 \%$. Dosage forms used were mostly oral (69.54\%). Infectious and parasitic diseases were the most common illnesses (16.01\%) followed by diseases of respiratory system. The most common drug groups prescribed were GIT, antimicrobials, antihistaminics, multivitamins and minerals. The incidence of poly- 
pharmacy was also common with maximum number of prescriptions (26.8\%) having 5 drugs per prescription. Prescription audit is an important measure to improve the quality of care afforded by the hospitals. Data generated on morbidity pattern coupled with current practices of treatment of these diseases provides an objective basis for preparing an NLEM. By this data we conclude that poly-pharmacy is quite common. Most of drugs were prescribed according to the NLEM 2011.

Dawit Kumilachew Yimenu et al., 2019 A total of 968 drugs were prescribed from 600 patient encounters. The average number of drugs per encounter was 1.6. The percentage of encounters in which an antibiotics and injections were prescribed was $69.7 \%$ and $6.3 \%$ respectively. Amoxicillin (28.5\%) followed by ciprofloxacin (12\%) and metronidazole $(11.1 \%)$ were the most commonly prescribed antibiotics. The percentage of drugs prescribed from essential drugs list and by generic name was $95.3 \%$ and $96 \%$, respectively. Rate of antibiotics prescribing showed deviation from the standard recommended by World Health Organization whereas polypharmacy, injectable prescribing pattern, uses of brand names, and prescription of drugs from the National Essential Drugs List were not found to be a significant problem though there were slight deviations from the standard. Interventions aimed at improving the antibiotic prescribing patterns need to be implemented so as to prevent the inappropriate use of antibiotics and avoid further complications.

Giorgia Sulis et al., 2020 the pooled prevalence proportion of antibiotic prescribing was $52 \%$ ( $95 \%$ CI: $51 \%-53 \%$ ), with a prediction interval of $44 \%-60 \%$. Individual studies' estimates were consistent across settings. Only 9 studies assessed rationality, and the proportion of inappropriate prescription among patients with various conditions ranged from $8 \%$ to $100 \%$. Among 16 studies in 15 countries that reported details on prescribed antibiotics, Access-group antibiotics accounted for more than $60 \%$ of the total in 12 countries. Antibiotics are highly prescribed in primary care across LMICs. Although a subset of studies reported a high proportion of inappropriate use, the true extent could not be assessed due to methodological limitations. Yet, our findings highlight the need for urgent action to improve prescription practices, starting from the integration of WHO treatment recommendations and the AWaRe classification into national guidelines.

A Y Massele et al., 1993 The percentage of patients leaving the dispensaries with no prescribed drugs was $1.3 \%$ and $0.7 \%$, respectively. Prescriptions containing antibiotics were 36.8070 (retrospective) and 39.8\% (prospective), while injections accounted for $24.6 \%$ and $34 \%$ of the total encounters, respectively. Over $70 \%$ of prescriptions conformed to the Tanzania essential drug list (EDP) and/or standard treatment guidelines and consisted of $83.9 \%$ and $79.1 \%$ generic prescriptions, respectively. Interestingly, only $15 \%$ of the surveyed dispensaries had an EDP book and/or calendar. Our study indicates that despite the adequate favourable performance of the dispensaries, as measured by the indicators, there existed variability between health facilities. We would like to stress the importance of such variability as a 'problem identifier' for planning interventions or supervisory actions. The lack of significant differences in the indicators 
measured retrospectively or prospectively is of practical importance. The limitations of poor record keeping in retrospective studies may be overcome with prospective surveys.

KAMBARALIEVA BAKTYGUL et al., 2011 a total of 19 different antibiotics were prescribed. Penicillin G (24.9\%), gentamicin (16.1\%), metronidazole (15.6\%) and cefazolin $(14.5 \%)$ were those most frequently prescribed. The major indications for antibiotics were diseases of the respiratory system (28.0\%), injury, poisoning and certain other consequences from external causes (25.5\%), and diseases of the digestive system (14.3\%). Almost threequarters of the antibiotics were used parenterally, 252 of which (58.9\%) were administered intramuscularly and $70(16.4 \%)$ intravenously. Forty-five percent of the patients received two antibiotics, and $12.0 \%$ received three antibiotics during their stay at the hospital. Antibiotic therapy proved inappropriate for 184 patients $(73.3 \%)$. The most common reason given for inappropriateness was the unjustified (not indicated) use of antibiotics in 143 (48.6\%) cases. There was a significantly higher inappropriate choice of antibiotics in gynecology $(\mathrm{OR}=2.70$, 95\% CI=1.02-7.69). We concluded that antibiotic prescriptions were seriously inappropriate in the Kyrgyz Republic with prescribing patterns failing to strictly adhere to the national guidelines. Adoption of an international standard and locally conformable guidelines of antibiotic use can help correct such problems.

Shikha Mishra et al., 2016 two Hundred and fifty prescriptions were analyzed. Total no. of drugs in 250 prescriptions was 1001. Therefore average number of drugs/ prescription is 4.004. Drugs were prescribed by generic names in $3.79 \%$ of cases, drugs on EDL are only $53.25 \%$ and fixed dose combinations are $26.87 \%$ of total drugs. Dosage forms used were mostly oral-93.51\%. Injectables were only $6.19 \%$ and topical forms were least $0.299 \%$. Basic information of patient was written in $72.57 \%$ prescriptions. Complete diagnoses were written in $70.04 \%$ prescriptions. Only $88.61 \%$ prescription were legible and only $76.79 \%$ prescriptions were complete in terms of dose, route, dosage form, strength and frequency. Most common drug groups prescribed were NSAIDs+ serratiopeptidases, antibiotics, antihistaminics, multivitamins, minerals enzymes and expectorants \& bronchodilators. This study showed that there is scope for improvement in prescribing patterns in areas of writing generic names of drugs, essential drugs, writing legible and complete prescriptions as well as writing prescriptions in capital letters. Polypharmacy was also evident from our study. Establishment and implementation of appropriate clinical guidelines, use of essential medicines list, public education about medicines and regular update to the clinicians will help in implementing the principles of rational pharmacotherapeutics.

M.H.Sumana et al., 2015 a total of 310 prescriptions of patients attending the Dermatology OPD were included for analysis. Among the 310 prescription studied 124 (40\%) were males \& 186 (60\%) were females. Among 310 Patients, majority were between the age group of 20-60 yrs. 945 drugs were prescribed, with an average of 3.04 drugs per prescription. Out of all drugs 92.6\% were from National essential drug list. Only 290(30.68\%) drugs were prescribed under generic names while brand names were used for 655(69.31\%) drugs. Drugs prescribed by parenteral route were $65(6.87 \%)$, oral route were $410(43.38 \%)$ and topical $470(49.73 \%)$. Out 
of all the topical agents, $94.2 \%$ were given as single preparations and $5.8 \%$ as combination agents. Antihistaminics were the most commonly prescribed group of drug. Periodic therapeutic audit is essential to ensure rational medicine use.

Sangita G. Kamath et al., 2014 among 464 antimicrobials prescribed to 154 patients, 13.79\% were prescribed by generic name, $73.28 \%$ were prescribed as parenteral preparations and 16 patients received single antimicrobial agent. Mean duration of hospitalization was $10.56 \pm 5.7$ days. Beta lactam antimicrobials apart from penicillins (J01D) were the most commonly prescribed. $78.88 \%$ of the prescriptions were approved by both DCGI and FDA. $72 \%$ of the prescriptions were adhering to the both NLEM and WHO-EML. $72.85 \%$ of the prescriptions were single drug formulations. 27.16\% were FDCs and 3 of them were not approved either by DCGI or FDA, nor included in any essential medicines list. Among prescriptions analyzed, cefoperazone + sulbactam FDC was not approved by the regulatory bodies nor included in essential medicines list. Majority of antimicrobials were prescribed by brand name. Results indicate that there is a scope for improving prescription pattern which can be facilitated by tailoring the hospital antimicrobial usage guidelines and monitoring the same.

Gurpreet Singh et al., 2016 A total of 4096 drugs were prescribed in 1200 prescriptions. Thus, an average of $3.41( \pm 2.07)$ drugs per encounter was prescribed. Generic name was used for prescribing $2008(49.02 \%)$ drugs. Antibiotics and injectable were prescribed in $222(18.5 \%)$ and 48 (4\%) prescriptions, respectively. Polypharmacy was seen in 286 (23.8\%) of prescriptions. Such detailed studies can contribute vital inputs for the development of evidence-based training modules for rational drug use even at the institutional level. Availability and accessibility of essential drugs at affordable prices can be achieved with rationale use of drugs.

Somenath Ghosh et al., 2016 Overall, an average number of drugs per prescription were $3.01 \pm 0.02$. Only $155(32.22 \%$ ) out of the total 481 drugs were prescribed by generic names. Among prescriptions antibiotics were in 71.87 percent and injectable preparations in 11.87 percent; 91.06 percent drugs were prescribed from national list of Essential Medicines of India. In terms of correctness, content of the drugs prescribed, was unacceptable; adherence to WHO core prescribing indicators was poor. Our study revealed that we need to identify loopholes regarding practices to improve the standard of prescriptions.

Mohammed Shakeel et al., 2013 Common age group was in between 21 to 30 years. Commonest cause of hospitalization was febrile illness. Average number of drugs per prescription was 4.64. Most preferred route was intravenous route. Antimicrobials were the most frequently prescribed drugs and ampicillin (29.73\%) was the commonest amongst them. All the cases were managed by empirical treatment. In most of the patient mono antimicrobial therapy was given. $40 \%$ drugs were prescribed by generic name. Dose of most of the drugs was inappropriate. We conclude that there is an urgent need for correction of some irrational approaches. 
Mohammad Afsana et al., 2013 The average number of drugs per prescription was 3.1 and no single drug was prescribed by generic name. Use of antibiotic (50\% of encounters) was frequent. Only 50.75\% drugs were prescribed from national essential drug list (EDL). Percentage of encounters with an antiulcerant, a NSAID and a multivitamin \& multimineral prescribed were $41.67 \%, 46.67 \%$ and $23.67 \%$ respectively. This study revealed some of irrational practices like poly pharmacy, overuse of antibiotics and vitamins, no use of generic names, and less prescribed from essential drug list. It is suggested that the periodic evaluation of prescribing practices at the healthcare facilities should be done by proper involvement of physician, nurse and pharmacist for the rational use of medicine.

\section{Result:}

Based on inclusion and exclusion criteria, 46 papers were taken up for this review. Data from these papers was extracted on the basis of characteristics of the area of the study, type of interventions, the main findings of the articles and general characteristics of the study such as publication year, study design, author's name etc. For further supplementation of information following websites were used for grey literature: the World Health Organization (W.H.O), Centre for Disease Control and Prevention (N.C.D.C) and Indian Council of Medical Research (I.C.M.R).

The reviewed studies on the Prescription audit were observed to be conducted in hospitals on various populations and have been categorized based on the hospital which study was done.

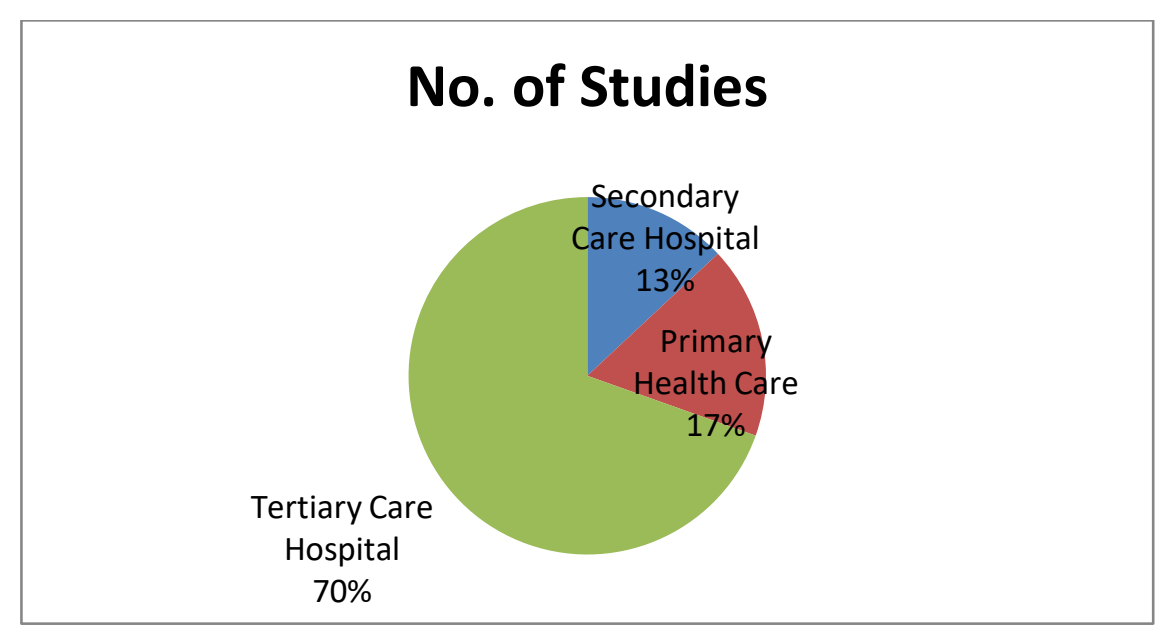

Figure 01 - Distribution of studies based on the type of hospital 


\section{ii) Type of Study:}

Based on our study on prescription audit of $\mathbf{4 6}$ papers major studies are Prospective study i.e. 16, followed by Cross-sectional study - 11, Retrospective study - 9, Survey based pilot study -3 and Observational study -7 .

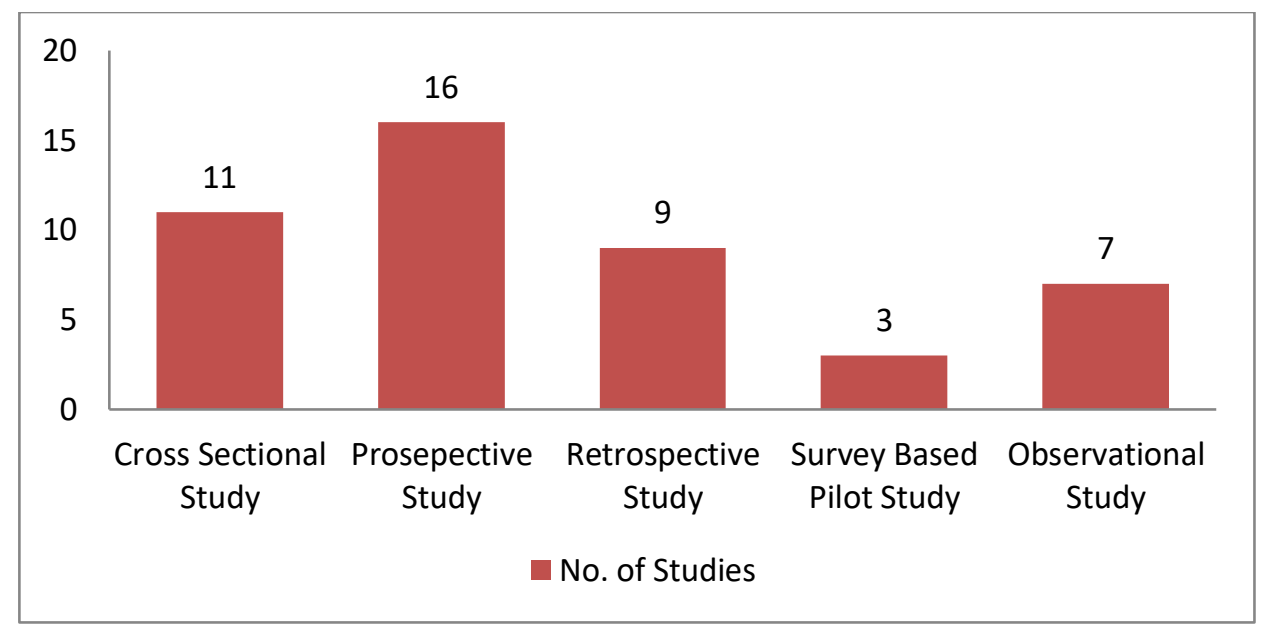

Figure 02: Types of study analysis

\section{iii) Prescription Audit conducted on Different Region of India:}

On basis of observation on time of literature review on prescription audit papers out of 46, major studies has been taken from Southern part of India i.e. 14, whereas 11studies has been taken from both Northern \& Western region of India. Least studies were included from Eastern region of India i.e. 10.

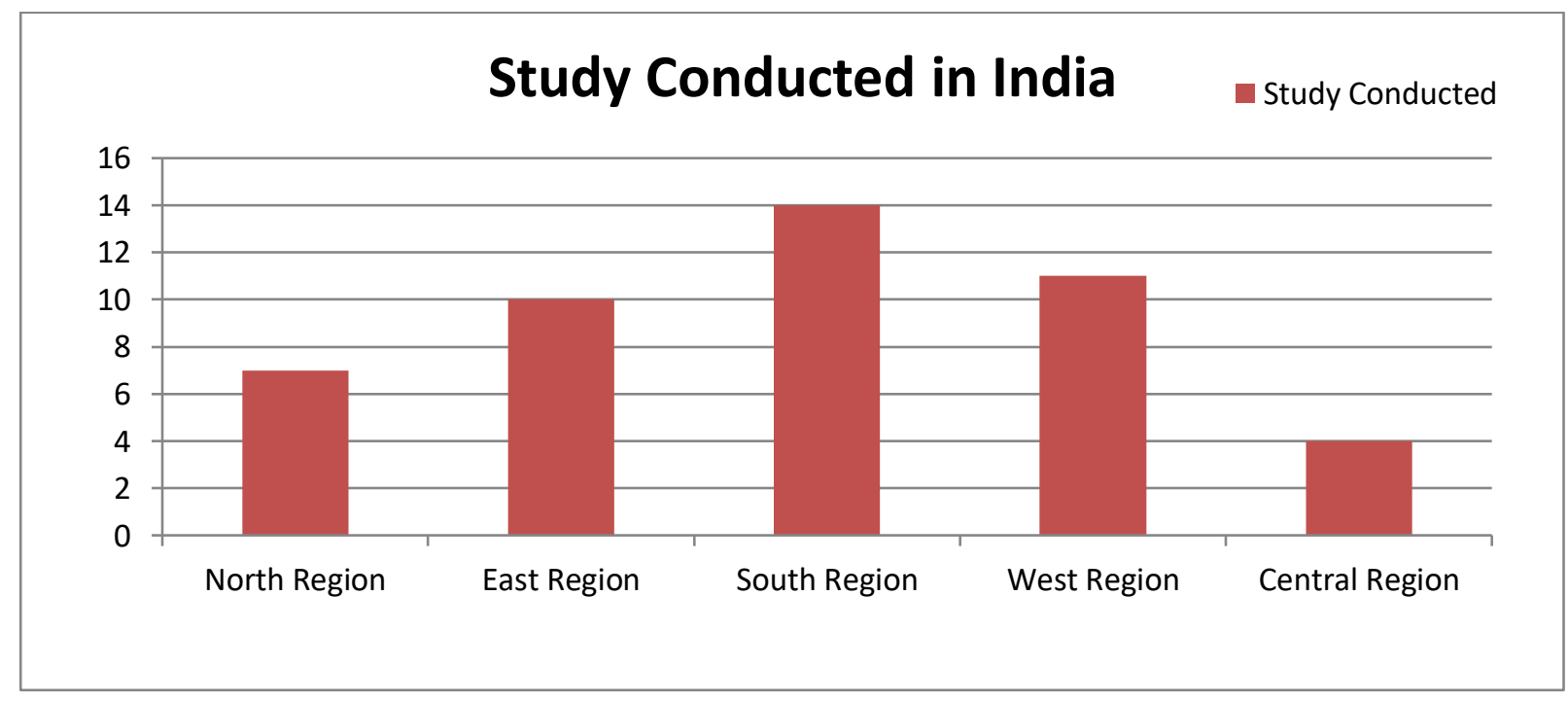

Figure 03: Region wise study conducted in India 


\section{iv) Total no. of studies in I.P.D and O.P.D:}

Based on our study on prescription audit of $\mathbf{4 6}$ papers major studies are conducted at OutPatient-Department (O.P.D) i.e. 80\% and 20\% conducted in In-Patient Department (I.P.D).

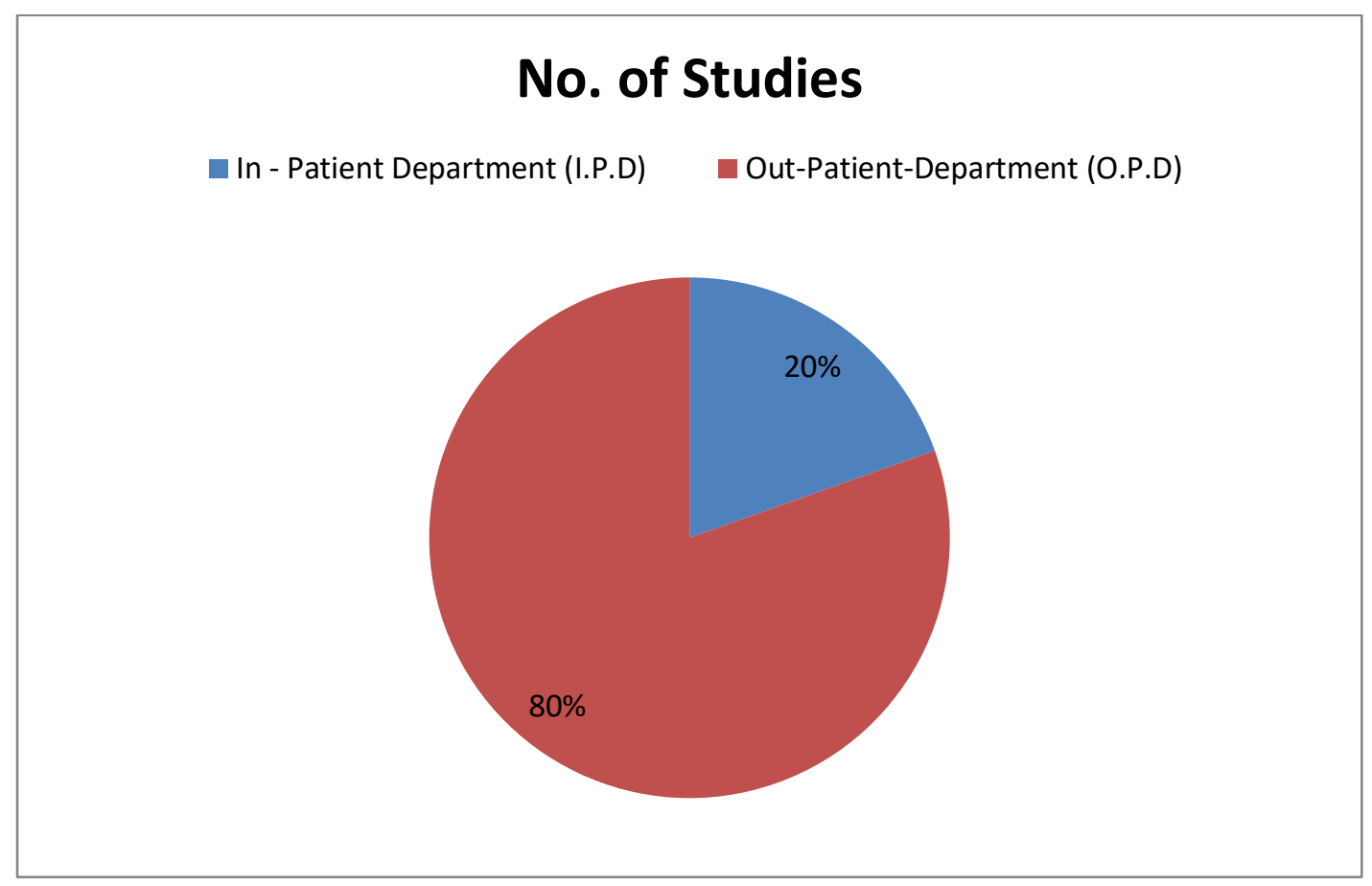

Figure 04: Place of Study - In Patient Department and Out Patient Department

This audit of the indoor patient prescriptions in various departments in the hospital was an eyeopening experience. The main objective was to assess the rationality of the prescription by the physicians in a hospital and to check whether they met the appropriate standards of good prescribing practices.

There have been limited studies conducted on prescription audits in India in the part of central region followed by north region of India. Conducting prescription audits frequently in hospitals and community pharmacies helps in the better understanding of the prescribing behavior of the physicians and reduces the risk of medication error by improving the quality of health care in these sectors.

This study showed that the highest no. of prescription audit conducted in the department of medicine followed by department of gynaecology, department of paediatrics at tertiary care hospitals of India (Figure 05: Department wise segregation at tertiary care hospital). 


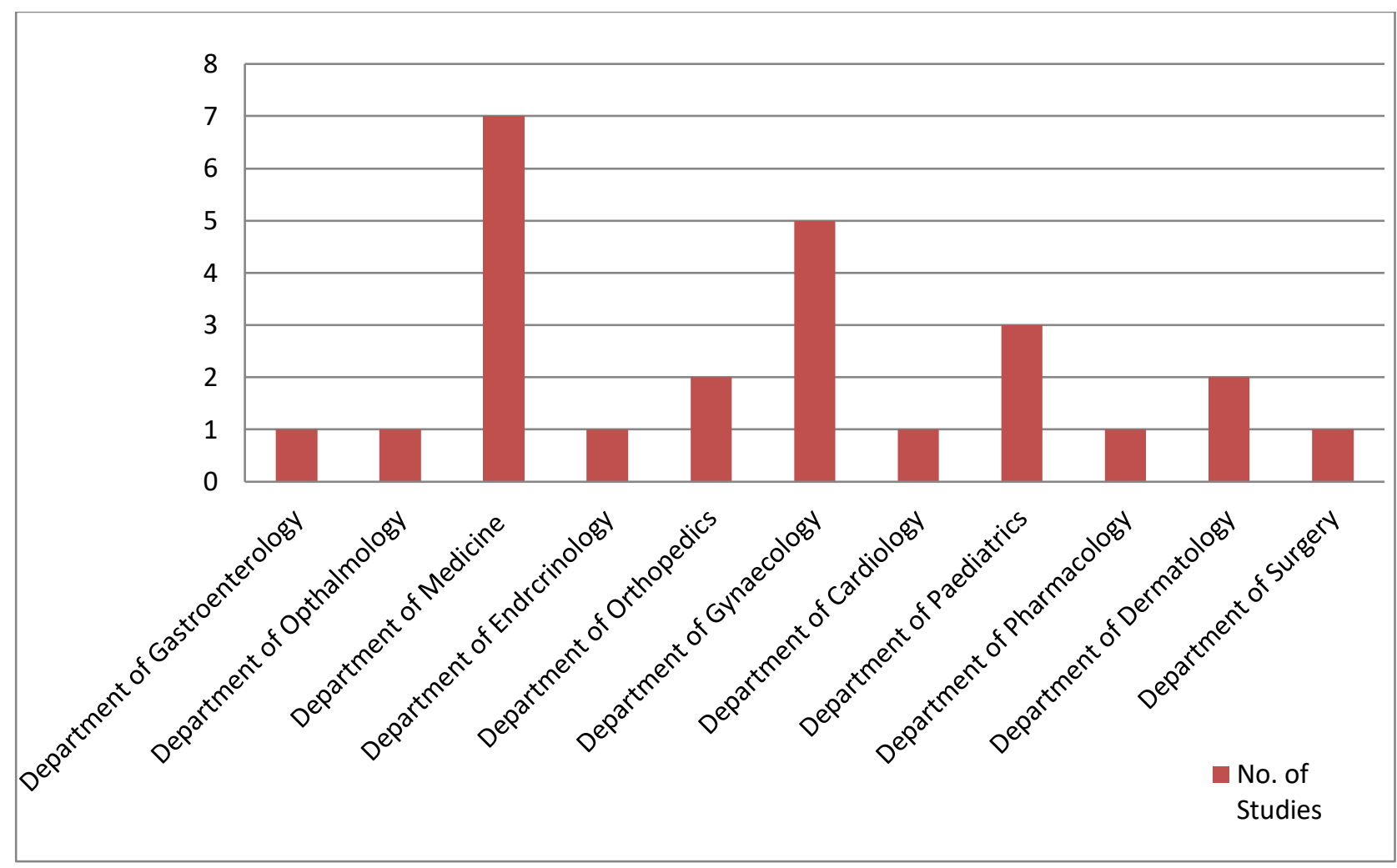

Figure 05: Department wise segregation at tertiary care hospital

\section{Conclusion:}

The studies shown that most of the practitioners are following the guidelines while writing the prescriptions. This would be a great starting if the standardization of prescribing patterns initiates in India so that all essential information is included and will be helpful for the better patient care. Some of the important component of the prescription (drugs should be prescribed in generic names and signature of the prescribing doctors should be in full, not just initial) should be properly addressed. All these measures should be helpful in providing optimal, low cost, and effective medicines to the patients. it is essential to choose right medicines and those should be administered in the appropriate manner, keeping the number of medicine as low as possible, using generic names, using the medicine appropriately after selecting consciously keeping the cost of the therapy low.

\section{References:}

1. A STUDY OF PRESCRIPTION AUDITING IN A TERTIARY CARE TEACHING HOSPITAL OF EASTERN INDIA, Journal of Drug Delivery \& Therapeutics; 2014, 4(1), 140-149,

2. Nirmal K Ganguly, Sujith J Chandy, Rationalizing antibiotics use to limit antibiotic resistance in India, Indian journal of medical research, 134, 2011, 142-154. 
3. World health organization, World health statistics, France; 2011.

4. kotwani A, Holloway K, Trends in antibiotic use among outpatients in New Delhi, India, BMC infect Dis., 11, 2011, 99.

5. Mercy ships bringing hope and healing. An essential medicines dosing guide based on the WHO model formulary 2008-Prescription writing guidelines

6. Fox A. Safer Prescribing, p1-23. Workbook-Section 1, Prescription writing.

7. Benet LZ. Goodman and Gilman's Principles of Prescription order Writing and Patient Compliance Instructions. The pharmacological basis of therapeutics. In: Gilman AG, Rall TW, Nies AS, Taylor P. 8th ed, vol II, New York: Pergamon press, 1991, 1640-49

8. Lewis VA. Prescription writing and Drug regulation. Pharmacology and Therapeutics for dentistry. In: Yogiela JA, Dowd FJ, Neidle EA. 5th ed., India: Mosby Publishers, 2005, 865-79.

9. A645 Prescription \& Non-Prescription Drug Guidelines Manual, Prescription \& NonPrescription Drug Guidelines.mht

10. Google Image Result for http--www_softchalk_com-lessonchallenge09lessonPharmacology-MC-pharmacy_medsManager_winter07_rxproof_1_jpg.htm, created with SoftChalk LessonBuilder

11. Teichman PG, Caffee AE. "Prescription writing to maximize patient safety". Fam Pract Manag 2002; 9:27-30.

12. Mikota SK, Plumb DC.Elephant Formulary. Abbreviations Used in Prescription Writing, Published by Elephant Care International - http://www. elephantcare.org, 2003-06.

13. Guidelines for Complete, Safe, and Accurate Discharge and Outpatient Prescription Writing, PTNews Virtual Hospital.htm, P\&T News: August 2003.

14. Shtrestha S. Irrational prescription: A hurdle to quality health. The Lancet 2013.

15. Kadam A. Rational Drug Use A Concern for Healthcare Professionals. Pharmainfo.net, 2009; 7: 4. 